\title{
Narrativa
}

Nuova serie

Italia fuori Italia

\section{Diverse Elena Ferrante}

\section{Sara Faccini}

\section{(2) OpenEdition}

\section{Journals}

\section{Edizione digitale}

URL: https://journals.openedition.org/narrativa/891

DOI: 10.4000/narrativa.891

ISSN: 2804-1224

\section{Editore}

Presses universitaires de Paris Nanterre

\section{Edizione cartacea}

Data di pubblicazione: 1 décembre 2016

Paginazione: 185-195

ISBN: 978-2-84016-266-7

ISSN: 1166-3243

\section{Notizia bibliografica digitale}

Sara Faccini, «Diverse Elena Ferrante», Narrativa [Online], 38 | 2016, online dal 01 janvier 2022, consultato il 22 février 2022. URL: http://journals.openedition.org/narrativa/891 ; DOI: https://doi.org/ $10.4000 /$ narrativa.891

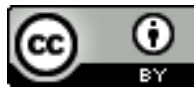

Narrativa est mise à disposition selon les termes de la Licence Creative Commons Attribution 4.0 International. 


\section{Diverse Elena Ferrante}

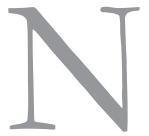

el presente saggio, mi propongo di evidenziare gli aspetti che contraddistinguono la ricezione dei romanzi di Elena Ferrante in Italia e negli Stati Uniti. Premetto che, leggendo le interviste rilasciate da Ferrante e gli articoli pubblicati in Italia e all'estero dedicati alla scrittrice e ai suoi romanzi, affiora un filo rosso o, meglio, affiorano due fili rossi: la volontà, affermata in più occasioni e con forza dalla scrittrice, di "raccontare con verità", di perseguire, attraverso la scrittura, una verità letteraria; e la volontà della critica, in particolare quella italiana, di scoprire chi sia veramente Elena Ferrante. Volontà quest'ultima a tratti ossessiva $\mathrm{o}$, quanto meno, esagerata.

In un'intervista rilasciata nel dicembre del 2015 al Financial Times Ferrante afferma: "Una pagina è ben scritta quando la fatica e il piacere di raccontare con verità hanno avuto la meglio su qualsiasi altra preoccupazione, anche la preoccupazione dell'eleganza formale. Appartengo alla categoria di chi butta via la bella copia e salva la brutta, se questa assicura maggiore autenticità"’; una convinzione che troviamo ribadita in un'altra intervista rilasciata recentemente alla prestigiosa Paris Review: ciò che muove la scrittura di Ferrante è "mettere in parole la verità di un gesto, di un sentimento, il flusso degli eventi, senza addomesticarli" Ferrante risponde:

1. Jobey, Liz, "Women of 2015: Elena Ferrante, writer", The Financial Times Magazine, 11 dicembre 2015. L'intervista è stata poi pubblicata in italiano: "Intervista a Elena Ferrante: i miei libri e l'enigma della mia vera identità", Il Sole 24ore, 23 dicembre 2015. Entrambe le versioni sono disponibili online.

2. Ibid.

3. Ferrante, Elena, "Elena Ferrante. Interviewed by Sandro and Sandra Ferri", The Paris Review. The Art of Fiction, n. 212, 2015. Consultabile online: http:/ / www.theparisreview.org/interviews /6370/art-of-fiction-no-228-elena-ferrante?src=longreads 
Per quanto mi riguarda, è il mio tormento e allo stesso tempo il motore di ogni progetto letterario. La questione più pressante è qual è la parola, qual è il ritmo della frase, [...]. La verità letteraria non è la verità di un biografo o di un giornalista, non è un rapporto di polizia o una sentenza emessa da un tribunale. La verità letteraria è $[. .$.$] direttamente proporzionale all'energia che uno è in$ grado di imprimere sulla frase. E quando funziona non c'è nessun cliché o stereotipo della letteratura popolare che vi resiste. Si rianima, sottopone tutto alle sue esigenze 4 .

Le parole di Ferrante rievocano il saggio Dire la verità di Natalia Ginzburg, la quale, nel 1935, scriveva:

Dire la verità. L'artista che scrive deve sempre sentirsi capace di questo. [...] L'artista non scrive una frase perché è bella, ma perché è vera. E non è un artista chi sacrifica la propria verità per amore di una bella frase o una bella parola. [...] Egli dipinge il suo mondo e i suoi personaggi quali sono, e non quali vorrebbero che fossero. Se no i personaggi sono falsi ${ }^{5}$.

Entrambe le scrittrici insistono sull'importanza di aderire alla realtà, alla verità della realtà, ritenendo che compito di chi scrive non sia cercare la bella frase, la bella copia, bensì lasciarsi guidare dalle vicende e dai personaggi in quanto solo riconoscendo piena autonomia ai personaggi e alle vicende raccontate si potrà scrivere ciò che davvero conta d'essere raccontato. Ciò che colpisce nella ricerca di verità qual è formulata da Ferrante è sentire riecheggiare, nei toni e rigore usati, la ginzburghiana coincidenza tra il piano estetico e quello morale, per cui una questione di poetica diviene una sorta di dovere morale nei confronti di lettrici e lettori ma, in primis, di se stesse.

La fedele e a tratti molesta adesione alla realtà, il puntuale seguire gli eccessi dei suoi personaggi attraverso una scrittura che, per usare le parole del critico letterario James Wood che nel 2013 consacrò il talento di Ferrante con un articolo sul New Yorker, "non ha limiti, spinge ogni pensiero verso la sua conclusione più radicale", sono tra le abilità di Ferrante che vengono evidenziate e

L'intervista è pubblicata in lingua inglese. Nostra la traduzione di questa citazione, come delle successive in originale inglese.

4. Ibid.

5. Cit. in Scarpa, Domenico, "Le strade di Natalia Ginzburg”, in Ginzburg, Natalia, Le piccole virtù, Einaudi, Torino 2015, p. XIII.

6. Wood, James, "Women on the verge. The fiction of Elena Ferrante", New Yorker, 21 gennaio 2013. Disponibile online: http://www.newyorker.com/ magazine/2013/01/21/women-on-the-verge 
generosamente lodate dalla critica, sia statunitense sia italiana, seppur con certe differenze. I critici e le critiche statunitensi e, più in generale angloamericani/e, discutono e riflettono sulla sua produzione, le tematiche ricorrenti e lo stile adottato, dedicando poco spazio alla scelta dell'anonimato, a differenza di critici italiani che raramente sembrano riuscire a interrogarsi sulla scrittura di Ferrante senza prima domandarsi chi sia e perché non voglia svelarsi.

Ma entriamo ora nel dettaglio. Fin dal suo esordio, con la pubblicazione nel 1991 di Amore molesto, Elena Ferrante esprime la ferma volontà di non fare nulla che comporti l'impegno pubblico della sua persona, come scrive in una lettera ai suoi editori Sandra e Sandro Ferri "[...] se il libro vale qualcosa, dovrebbe essere sufficiente. [...] Io credo che i libri non abbiano alcun bisogno degli autori, una volta siano stati scritti" ${ }^{\prime}$. Intenzione che, seppur mossa da ragioni diverse, viene ribadita un ventennio dopo nell'intervista alla Paris Review: la scrittrice racconta di aver "iniziato a sentire ostilità verso i media che $[\ldots]$ valorizzano un'opera a seconda del nome dell'autore. Come se la letteratura non fosse in grado di dimostrare la sua serietà semplicemente attraverso $i$ testi ma richie-

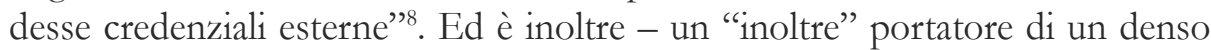
significato e di un chiaro posizionamento - importante sottolineare il fatto che Elena Ferrante abbia deciso di rappresentarsi come scrittrice e di adottare un punto di vista femminile e lo abbia fatto, come lucidamente evidenzia Tiziana de Rogatis, "[i]n un paese come l'Italia, in cui la casta maschile del giornalismo, dell'editoria e dell'accademia impedisce la visibilità (e direi anche il rispetto) delle nostre scrittrici, [...] [scegliendo] di essere una di loro" e dunque affermando "sul piano comunicativo (pronominale, sintattico, linguistico, tematico) e sul piano sociale che lo sguardo di una donna è decisivo" 10 .

Riflettendo oggi su tale scelta, non è da escludere che all'inizio l'anonimato possa aver ostacolato la popolarità e le vendite, ma a poco a poco il segreto identitario può aver favorito la curiosità e l'interesse, e di conseguenza il successo sia in Italia sia all'estero. Sta di fatto che, tenendo conto dello scarso numero di autrici e autori stranieri tradotti in inglese, il successo riscosso dai romanzi di Elena Ferrante negli Stati Uniti è eccezionale. I libri di Ferrante - sia

7. Ferrante, Elena, La frantumaglia, e/o, Roma, 2003, pp. 9 e 10.

8. Ferrante, Elena, "Elena Ferrante. Interviewed by Sandro and Sandra Ferri", cit.

9. De Rogatis, Tiziana, "Scegliere il punto di vista di una donna. Sul presunto smascheramento di Elena Ferrante", Nazione Indiana, 5 ottobre 2016. Disponibile on line: https://www.nazioneindiana.com/2016/10/05/ scegliere-punto-vista-donna-sul-presunto-smascheramento-elena-ferrante/

10. Ibid. 
la tetralogia dell'Amica geniale sia i primi tre romanzi brevi - hanno ottenuto apprezzamenti entusiastici da parte di critici e critiche letterarie di molti dei maggiori giornali, riviste e siti on line britannici e statunitensi: il New York. Times inserisce l'ultimo volume della tetralogia nella sua lista dei libri del 2015; il Guardian lo definisce "un romanzo da premio Nobel"; il Financial Time include Elena Ferrante nella lista delle donne del 2015, mentre The Paris Review dedica un'intervista all'autrice, come, prima di lei, solo ad altri quattro italiani, tutti uomini: Italo Calvino, Primo Levi, Alberto Moravia e Umberto Eco.

Il critico del New Yorker James Wood biasima l'indiscrezione della stampa italiana a intromettersi nella privacy di Ferrante, comprendendo e sostenendo la scelta della scrittrice: "Tale scelta - scrive Wood - [è] una forma di protezione. I suoi racconti sono violentemente, intensamente personali. [...] Sembra quasi che facciano balenare davanti agli occhi del lettore che nulla sospetta una serie decisiva di confessioni" 11 . Secondo Wood il contenuto "è schietto in modo sconvolgente: maltrattamenti infantili, divorzio, maternità, la voglia di avere o non avere figli, la noia del sesso, il ribrezzo del corpo, la lotta disperata della voce narrante per mantenere una solida identità all'interno di un matrimonio tradizionale" ${ }^{\prime 2}$. Vicina all'analisi di Wood è la critica Michiko Kakutani che sul New York Times, recensendo la tetralogia napoletana, evidenzia l'abilità di Ferrante nel creare

una sorta di corrente elettrica che dà ai romanzi una tensione narrativa coinvolgente. [...] Come Munro e Doris Lessing, Ferrante cattura la trama quotidiana della vita delle donne: lo sforzo di mantenere un po' di senso di sé nonostante le incessanti, banali incombenze domestiche - i pannolini e la cucina da pulire e spolverare - e le richieste di tempo e attenzione da parte di mariti e amanti ${ }^{13}$.

Katrina Dodson affianca a Ferrante non solo Munro e Lessing ma anche Clarice Lispector in quanto entrambe le scrittrici, afferma, raccontano "con grande originalità e verità che può colpire come un pugno allo stomaco. Mi

11. Woon, James, "Women on the verge. The fiction of Elena Ferrante", cit.

12. Ibid.

13. KaKUTANi, Michiko, "Elena Ferrante's 'The Story of the Lost Child', the Finale in a Quartet", New York Times, 3 settembre 2015. Disponibile online: http://www. nytimes.com/2015/09/04/books/review-elena-ferrantes-the-story-of-the-lost-childthe-finale-in-a-quartet.html?_r $=0$ 
sono sentita alternativamente sedotta e intrappolata dalle due autrici, che avvolgono il lettore nei mondi interiori dei personaggi" ${ }^{14}$.

In un interessante articolo, la critica statunitense Megan O'Rourke riconduce la profondità dei temi indagati e lo stile adottato da Ferrante nel trattarli all'assoluta libertà datale dall'anonimato. E a proposito della convinzione della critica del Guardian, possiamo ricordare che la stessa Heilbrun, in Scrivere la vita di una donna, riflettendo sui motivi che la spinsero a firmare come Amanda Cross i gialli che pubblicava, evidenziava come "l'anonimato [le] desse una sensazione di controllo sul proprio destino" in quanto "segretezza significa potere" ${ }^{15}$. Nel suo articolo O'Rourke, dopo aver rintracciato nei romanzi di Ferrante aspetti simili a quelli evidenziati dai già citati Wood e Kakutani - quali la scrittura fortemente intima e il riuscire a calare la lettrice e il lettore in un mondo completamente plasmato dalla percezione della narratrice - pone l'accento sull'abilità della scrittrice napoletana nell'indagare, attraverso "[una] giustapposizione tra il piano concreto e quello simbolico", il modo in cui "le donne vengono modellate, distolte e talvolta distrutte dal loro ambiente sociale (e dagli uomini che le circondano)" $"$.

Sempre riguardo il successo riscosso negli Stati Uniti, un aspetto importante che va sottolineato è il ruolo della traduttrice, la giornalista del New Yorker Ann Goldstein, lodata per la sua traduzione ineccepibile: secondo alcuni, la sua versione sarebbe persino migliore dell'italiano di Ferrante. In un articolo apparso sulla Rivista Quarts, Annalisa Marelli, giornalista italiana che vive a New York, sostiene che la disparità di giudizio tra l'entusiasta critica statunitense e la più fredda italiana sia dovuta proprio a questo, "alla diversa qualità di scrittura tra il testo italiano e quello inglese, [che] attenua molti limiti del linguaggio originario" ${ }^{17}$. Secondo Marelli la versione originale dei libri di Ferrante presenta "pochezza lessicale, $[\ldots]$ costruzioni casuali, $[\ldots]$ un tono incoerente e $[\ldots]$ una scrittura sempre forzata" 18 , a differenza della traduzione inglese in cui il testo è più scorrevole e il lessico meno pesante e artificioso.

14. "Katrina Dodson interviews Ann Goldstein", Guernica - a Magazine of Art \& politics. The Face of Ferrante, 15 gennaio 2016.

15. Heilbrun, Carolyn G., Scrivere la vita di una donna, La Tartaruga, Milano, 1990 [ed. orig.: 1988], pag. 143.

16. O'Rourke, Megan, "Elena Ferrante: the global literary sensation nobody knows", The Guardian, 31 ottobre 2014. Consultabile online: https://www.theguardian.com/books/2014/oct/31/elena-ferrante-literary-sensation-nobody-knows

17. Marelli, Annalisa, "Elena Ferrante's writing is better in English than Italian", Quartr, 16 dicembre 2015.

18. Ibid. 
Da un esame degli articoli fino a ora citati emerge un accordo: della produzione ferrantiana, a partire da I giorni dell'abbandono - il più letto in inglese - fino alla tetralogia dell'Amica geniale, ciò che appassiona maggiormente sono la voce e lo sguardo con cui la scrittrice riesce a mostrare la quotidianità familiare e domestica come una realtà infernale, piena di rancore e violenza, intrecciando e aggrovigliando la sfera pubblica a quella privata, una specchio dell'altra ed entrambe dominio maschile la cui forza, per riprendere Bourdieu, si misura dal fatto che non deve giustificarsi ${ }^{19}$. Come scrive Ambra Pirri in un interessante saggio dedicato all'ambivalenza in Elena Ferrante,

[n]el rione i corpi maschili "agiscono" la propria sessualità in piena e assoluta libertà, legittimati a tutto. Anche le ragazze vengono addomesticate attraverso il corpo ma, al contrario dei maschi, loro la testa la debbono abbassare insieme allo sguardo [...]. Se per le donne il corpo è un peso difficile da portare, per i ragazzi è esattamente il contrario: il loro corpo, invadente e minaccioso, può muoversi a piacimento, e l'uso che ne fanno insieme all'uso che fanno dei corpi femminili è una prova di mascolinità che costantemente si scambiano, mostrando così che il legame sesso-potere è la struttura fondante dell'ordine maschile $e^{20}$.

Oltre alla capacità di interrogare quei nodi esistenziali che ci coinvolgono e interrogano intimamente - per citare solo i più importanti: il rapporto con la madre e con il suo e il nostro corpo, la maternità, il terrore dell'abbandono da parte del marito o amante che sia e i continui compromessi cui arriviamo, senza neppure troppo dolore, nella vita che decidiamo di condurre - qual è l'aspetto che più affascina il lettore e la lettrice statunitensi del mondo ferrantiano? Forse proprio il suo "essere un mondo"?

È di quest'idea Laura Buffoni che accosta la scrittrice napoletana a Jonathan Franzen: l'una autrice, l'altro autore di "romanzi-vite" nei quali è tutto codificato ma allo stesso tempo - e penso stia qui l'abilità - tutto autentico: "ciò che di veramente interessante (e insidioso) fa Ferrante è costruire un mondo artificiale $[\ldots]$ per poi aprirlo alla flagranza del reale" ${ }^{\prime 2}$. Un mondo fatto di ingiuste regole codificate e improvvise rotture, ossia la vita. E allora viene da doman-

19. Bourdieu, Pierre, Il dominio maschile, Feltrinelli, Milano 1998 [ed. orig.: 1998], pag. 45.

20. PIRri, Ambra, "Elena Ferrante: nomi e corpi ambivalenti", in Crispino, Anna Maria, Vitale, Marina (a cura di), Dell'ambivalenza. Dinamiche della narrazione in Elena Ferrante, Julie Otsuka e Goliarda Sapienza, Iacobelli editore, Roma, 2016, pp. 60-61.

21. Buffoni, Laura, "Elena Ferrante sono io", Internazionale, 3 novembre 2014. 
darsi se ciò che soddisfano le pagine di Ferrante non sia il desiderio di raccontare la storia di Olga, Leda, Lila e Lenù, quanto la nostra storia. Nadia Terranova, in un articolo in cui si interroga su chi siano i "consacratori americani di una serie tutta napoletana" 22 , evidenzia la varietà del pubblico di Ferrante e come "ciascun lettore [sia] convinto che lei scriva esclusivamente per lui" 23 . Nei romanzi di Ferrante si realizza una peculiarità della letteratura, ossia la capacità di narrare a lettrici e lettori la loro storia, rivelando la loro unicità. Adriana Cavarero, nell'illuminante $T u$ che mi guardi, tu che mi racconti, definisce la narrazione un'arte delicata in quanto - utilizzando le parole di Hannah Arendt - "rivela il significato senza commettere l'errore di definirlo": "l'accidentale che è in ogni vita" ha bisogno di essere salvato o, meglio, scrive Cavarero, "ha bisogno di cura":

$[R]$ accontare la storia che ogni esistenza si lascia dietro è forse il gesto più antico di tale cura. Non necessariamente una storia che aspiri a immortalarsi nell'empireo letterario ma piuttosto il tipo di storia il cui racconto si appaesa persino negli angoli delle cucine, davanti a un caffè, oppure sul treno, [...e e] sono sintomaticamente soprattutto le donne a raccontare storie di vite ${ }^{24}$.

Donne narratrici eccellenti del particolare, un "particolare splendente di finitezza e pago del suo esistere, la cui gloria prende [...], per gli umani, la forma dell'unicità' 25 .

Sul numero di novembre 2014 di Leggendaria, Anna Maria Crispino apre il suo articolo scrivendo: "Se in Italia avessimo l'ossessione del 'grande romanzo nazionale' come negli Usa, dove si cerca di scrivere (e di leggere) il 'grande romanzo americano', forse potremmo affermare che Elena Ferrante ci è riuscita" 26 . Leggendo Crispino a proposito dell'atteso disatteso "grande romanzo americano", penso a ciò che sostiene la giornalista newyorkese Alissa Quart, la quale, affascinata in particolare dall'importante ascesa sociale della protagonista della tetralogia Elena Greco, si domanda dove siano oggi i romanzi

22. Terranova, Nadia, "Vendere all'estero un'amica geniale", Rivista Studio, n. 24, 2 luglio 2015. Disponibile online: http://www.rivistastudio.com/standard/vendere -allestero-unamica-geniale/

23. Ibid.

24. Cavarero, Adriana, Tu che mi guardi, tu che mi racconti. Filosofia della narrazione, Feltrinelli, Milano, 1997, p. 73.

25. Ibid., pag. 72 .

26. Crispino, Anna Maria, "L’una e l'altra”, Leggendaria, n. 108, novembre 2014, p. 48. 
sociali americani che raccontano le fluttuazioni economiche, la mobilità sociale e le diseguaglianze sociali nella maniera intensa in cui l'ha fatto Ferrante per l'Italia. Quart esprime il timore che i romanzi di carattere sociale siano stati penalizzati prima dal minimalismo e poi dai miraggi di arricchimento individuale, alimentati dai programmi televisivi dalle ricchezze facili. E con amarezza Quart conclude scrivendo: "E io - e noi - dobbiamo rivolgerci alla Napoli romanzata di Ferrante per leggere la storia che vogliamo credere ancora possibile nel nostro paese e incontrare un orizzonte di donne che crescono, cadono e si rialzano" ${ }^{\prime 27}$.

Oltre ai motivi fino a ora individuati che possono aver affascinato i lettori e le lettrici d'Oltreoceano, ci si può chiedere se sul successo di Elena Ferrante all'estero non abbia inciso la rappresentazione di una realtà napoletana e italiana a tratti stereotipata. Per esempio, il modo in cui Ferrante, nella tetralogia, attraversa il Novecento, può lasciar perplessi: potrebbero non convincere il suo farlo abitare da personaggi-tipo - quali, per citarne solo alcuni: l'arrampicatore sociale e latin lover Nino che con leggerezza colleziona donne sedotte e appartenenze politiche, o i componenti della famiglia Airota, esponenti dell' intellighenzia fiorentina - e non convince neppure il suo citare in maniera vaga nodi molto importanti della storia italiana, come la scelta della lotta armata, i percorsi d'autocoscienza di alcuni gruppi femministi o la diffusione dell'aids, e le conseguenze politiche che ne derivarono.

A mostrare che quel modo di attraversare il secolo ha sue interessanti peculiarità sono le acute analisi di intellettuali quali le già citate Pirri, Crispino e de Rogatis e con loro Fortini, Pigliaru, Brogi e Terragni. Osserva Marina Terragni che le lettrici sono aiutate nellidentificazione "dal fatto che Ferrante nasconde il suo volto [e] lo scambio tra lei che scrive senza mostrarsi e te che leggi è quasi alla pari, [e] la storia non è di nessuna e perciò è di tutte"28; Fortini evidenzia che le donne e gli uomini vivono "in anni scanditi da date certe, non quelle della Storia grande, ma quelle della vita delle due protagoniste"29, perché Ferrante non intende dare un'interpretazione degli anni Settanta ma mostrare come "la dimensione pubblica e quella privata sono in ogni caso, e in ugual misura, riconducibili in primissima istanza a quelle due singole vite" ${ }^{30}$. E nota Pirri: "Non è

27. Quart, Alissa, "Why Doesn't America Have An Elena Ferrante?”, BurzFedd Books, 10 novembre 2015.

28. Terragni, Marina, "La scrittrice geniale", Io Donna, 14 gennaio 2012.

29. ForTini, Laura, "Due ragazze del secolo scorso raccontate da Ferrante", Il Manifesto, 31 ottobre 2012.

30. Crispino, Anna Maria, "Il gioco del doppio", Leggendaria, n. 103, gennaio 2014. 
certo sul Novecento che riflette la scrittrice napoletana, certamente lo attraversa ma lo lascia sullo sfondo insieme agli uomini; forse è per questo che il contesto è superficiale, è come gli uomini che lo hanno determinato" 31 . Non credo sia una coincidenza che le intellettuali citate siano anche femministe e adottino un punto di vista di genere nella loro interpretazione.

Perché invece, in Italia, i critici si sono mostrati molto più freddi nei confronti di Elena Ferrante? Escluso Goffredo Fofi e pochi altri che hanno affiancato la scrittrice a grandi nomi della letteratura tra cui quello di Elsa Morante, i più hanno manifestato forti perplessità o si sono limitati a discutere le ragioni dell'anonimato, senza entrare nel merito della scrittura o criticandola. Francesco Longo, riferendosi a Storia di una bambina perduta, afferma che

lo stile è assente. Gli accostamenti linguistici sono abusati e il libro risulta scritto in una lingua di nessuno. [...] le psicologie dei personaggi risultano abbozzate [...] Tutto è psicologicamente piatto [...]. In mancanza di stile le passioni si accendono in modo artificioso e piatto, $[\ldots]$ le banalità, in quindici pagine, non si contano ${ }^{32}$.

Filippo La Porta vede nell'anonimato di Ferrante una "smisurata volontà di potenza" 33 e confessa che, dopo averla amata nei primi romanzi, "con il ciclo dell' Amica [ha] smesso di seguirla, forse perché un po' respinto da questo narcisismo letterario che, tra l'altro, è andato di pari passo a un appiattimento della lingua" 34 . L'articolo di La Porta si intitola "Su Elena Ferrante. Un gioco è bello quando dura poco", e diversi critici restano impegnati nel giocare a domandarsi chi si nasconda dietro lo pseudonimo di Elena Ferrante.

Più sorprendenti sono le espressioni misogine di Sebastiano Vassalli che, adirato dalla candidatura della scrittrice al Premio Strega, la definisce "una donnarella tremebonda", una "personcina qualunque", augurandosi che il premio lo vinca qualcuno "con un nome certo e una storia su cui si reggono le storie che racconta" 35 . Altrettanto virulento è Paolo di Paolo, secondo il quale i critici americani amano Ferrante "per le sue trame oliate, la lingua piana, e Napoli

31. PIRRI, Ambra, "Elena Ferrante: nomi e corpi ambivalenti", cit., p. 72.

32. Longo, Francesco, "Troppa importanza all'amore, poca importanza all'amore”, Rivista Studio, 25 maggio 2015.

33. La PORTA, Filippo, "Su Elena Ferrante. Un gioco è bello quando dura poco", Wikicritics, 14 marzo 2016.

34. Ibid.

35. Vassaldi, Sebastiano, Corriere della sera, 31 marzo 2015. 
[che] sta lì come una stampa turistica" ${ }^{36}$, mentre quelli italiani "se fosse un'altra autrice - una che $[. .$.$] 'ci mette la faccia" { }^{37}$ - sarebbero più severi", sicuro che "la forza di Ferrante è, più che nei suoi libri, nel suo non esserci" 38 .

Romano Luperini dice di non essere riuscito a concludere Storia di una bambina perduta sentendosi "schiacciato in un tritarsi di infiniti piccoli fatti e di infiniti personaggi che si susseguono incessantemente senza che nessuno di essi acquisti un qualche rilievo linguistico, stilistico o tematico. [...] Tutto viene troppo 'detto' e poco rappresentato" 39 . Tuttavia Luperini, considerati i giudizi positivi su Ferrante espressi da colleghe da lui molto stimate, si chiede "se nel [suo] fastidio non ci sia una dose di sessismo", e invita critici e critiche a confrontarsi apertamente.

Quale conclusione trarre da questa rapida carrellata comparativa? Se pensiamo a frasi come "donnarella tremebonda" o "le banalità non si contano", o alla volontà di limitare l'interrogativo alla misteriosa identità di Ferrante, viene proprio da chiedersi, come fa Luperini, se non persista una dose di sessismo presso molti intellettuali nostrani, in continuità con la tradizione critica italiana. E sul punto, basti ricordare le espressioni usate da Cesare Garboli per definire la scrittura di Natalia Ginzburg, caratterizzata da una "incosciente infrazione dei codici" ${ }^{40}$ e da un "punto di vista femminile, oscuro, viscerale, primitivo, diverso, uterino" ${ }^{41}$; ora, quella scrittura nulla aveva di incosciente, eppure restava per lui viscerale, oscura e uterina, caratteri connaturati al femminile.

In un bell'intervento tenuto alla Biblioteca delle Donne di Bologna, dal titolo "Critica e Genere: Elena Ferrante e Alba de Céspedes", Tiziana de Rogatis ha

36. Di Paolo, Paolo, "Il caso Ferrante. Il romanzo italiano secondo il New Yorker", La Stampa, 13 ottobre 2014.

37. A proposito del "metterci la faccia" di cui scrive Di Paolo, mi permetto di rinviare al già citato articolo di Tiziana de Rogatis la quale, in merito alle continue e anche recenti indagini circa la vera identità di Elena Ferrante evidenzia che "la fame di realtà è un bisogno legittimo che muove le nostre esistenze ma non potrà mai essere soddisfatto dalla violazione della vita altrui"; cfr. https://www.nazioneindiana.com/2016/10/05/ scegliere-punto-vista-donna-sul-presunto-smascheramento-elena-ferrante/.

38. Ibid.

39. Luperini, Romano, “L'ultimo libro di Elena Ferrante: discutiamone. Il caso Ferrante/1", Blog Letteraturaenoi, 20 aprile 2015. Il post è stato ripubblicato di recente (6 agosto 2016) sul medesimo blog, al fine di rilanciare il dibattito proprio sul punto dell'apprezzamento condizionato dal gender; cfr. http://www.laletteraturaenoi.it/ index.php/interpretazione-e-noi/349-l-ultimo-libro-di-elena-ferrante-discutiamone. $\mathrm{html}$

40. Garboli, Cesare, "Fortuna critica", in Ginzburg, Natalia, Opere, II, Mondadori, Milano, 2001, p. 1590.

41. Garboli, Cesare, "Prefazione", in Ginzburg, Natalia, Opere, I, cit., p. XXV. 
riflettuto, a partire dai due casi della scrittrice napoletana e della scrittrice italocubana, sulla fatica delle donne a essere considerate al pari dei colleghi uomini. E circa l'ipotesi sostenuta da molti che dietro Elena Ferrante ci sia uno scrittore uomo, la studiosa si domanda se tale ipotesi sia "sintomatica di una più generale rimozione italiana della presenza e autorevolezza femminile" e quanto "per l'immaginario collettivo sia rassicurante ipotizzare che una delle scrittrici più famose sia scritta [sic] da un genio maschile" ${ }^{\text {"2 }}$.

Vogliamo sperare che Tiziana de Rogatis venga confutata, che non sia rassicurante pensare che dietro Elena Ferrante si nasconda un genio maschile. Per ora constatiamo questo: a differenza della critica italiana, la critica americana poco si interessa all'identità sessuale di Ferrante e soprattutto non usa categorie viete: niente "donnarelle tremebonde", punti di vista uterini o forza genitale ${ }^{43}$.

Sara FACCINI

Centre de Recherches Italiennes (CRIX- EA369 Études Romanes), Université Paris Nanterre e Sapienza-Università di Roma

42. De Rogatis, Tiziana, "Critica e Genere: Elena Ferrante e Alba de Céspedes", intervento tenuto alla Giornata di studio Davanti e dietro la scrittura. Donne e uomini alle prese con identità di genere, ruoli, gerarchie e riconoscimento pubblico, Biblioteca delle Donne, Bologna, 18 aprile 2015, disponibile su https://vimeo.com/128848293.

43. Mentre il presente articolo era in corso di stampa, un'inchiesta condotta da $I l$ Sole 24 Ore e pubblicata dal quotidiano Frankfurter Allgemeine Zeitung, dal sito francese Mediapart e dalla rivista statunitense The New York Review of Books, afferma di aver accertato l'identità della scrittrice. Tale "scoperta", oltre ad aver ricevuto smentita, non inficia le tesi qui esposte. 
